\title{
Virtual Ergonomics Laboratory: Human Body Dimension Relevance
}

\section{Debkumar Chakrabarti ${ }^{1 . a}$, Manoj Deori, Sangeeta Pandit and Ravi T}

\author{
${ }^{1}$ Department of Design, \\ Indian Institute of Technology Guwahati, Guwahati 781039, Assam, India \\ adc@iitg.ernet.in
}

Keywords: Virtual ergonomics laboratory, physical human dimensions, animated experiments

\begin{abstract}
Ergonomics has become an integral part of design education curriculum, where input content demands demonstration through citing and analysing appropriate design experiences. It has come to fore through many academic forum discussions and meetings that to internalise various ergonomics issues relevant hands on experiences on this are necessary. To impart a feel of laboratory experimentation as well as application relevance to a greater number of learners, a virtual environment scenario could go along.

A virtual presentation of ergonomics laboratory experiments on physical anthropometry and its design dimension consequences has been tried out. It contains a total of eleven sections. The topic opens with the introductory session where the subject matter and the laboratory experiment methodology in general was considered; this was followed by ten specific topics with flash based self-learning modules and data support on Indian population was provided to have a ready reference. Some of these topic specific experiment sections are also backed with video demonstrations.

A whole set of virtual laboratory module under development, for users' feedback, has already been uploaded in the net. The experiments are self-explanatory, downloadable and easy to perform. The feedback collected so far (online and also through direct demonstration surveys), confirms its usefulness both by the teachers and student-learners of Ergonomics specialisation and design programmes. This paper reports the salient features with content outline of the educational and free to use virtual anthropometric experiments manual developed which is being fine-tuned at IIT Guwahati.
\end{abstract}

\section{Introduction}

Ergonomics has become an integral component of design and engineering curriculum in India and to meet the requirement trained man power and laboratory facilities is too far from reaching the required level. As an outcome of various levels of academic meetings and forums, a need to go for non-contact and using the benefits of electronics media input was felt. Thus the very intention to develop a virtual ergonomics laboratory setup to provide a free to all academic input was conceived and executed. In design, most of the engineering fields, architecture and management education including occupational health and safety though ergonomics is being considered as one of the core inputs, is yet to get proper impetus, and mostly the requirement is met with improper attention though its importance is widely talked about.

Academicians and expert practitioners feel the general reference somehow available in various literature sources do not meet the expected need and most of the references are based on non-India sources. Input facility of ergonomics is as such inadequate in India and well equipped ergonomics laboratories are very few and even that all laboratories also do not have all the facilities. Many design, architecture and engineering students have mentioned that they are aware about the subject and they need easy/ ready source on the subject matter and hands-on experiences with India specific references [1]. Discussions with academicians reveled that discipline relevant hands-on practical experimentation would have a desired effect, but to start with providing a basic level input would be good; the present project is an initiation with human physical compatibility issues in general through virtual approach, to serve an additional supplement to the direct contact teaching-learning. Thus the 'Ergonomics Lab for Assessing Physical Aspects of Design' (a tutorial manual) exercise, conceived by ergonomics laboratory of IIT Guwahati is an effort to make the techniques familiar 
for applying and measuring ergonomics interfaces relevant to the humanising technology; specifically in this current effort it concerns with human physical dimensions relevance. This development was conceived as a component project of a nationwide virtual laboratory for technical education initiative. This paper reports its content out line of ergonomics component.

\section{Objectives}

This approach aimed at developing a self-learning tutorial material with inbuilt know-how demonstration and laboratory techniques of measuring basic anthropometric dimensions and some human compatible physical interface in the man-machine-environment system interaction.

It worked upon specific objectives:

1. To make interactive simulation based laboratory manual for measurement of anthropometric aspects of engineering and design relevance; and

2. To see the effectiveness of such packages in context specific ambient with specific reference to Indian anthropometric data support.

\section{Approach and concept of imparting virtual ergonomics laboratory experience}

The virtual laboratory base for the relevant experiments developed herein to have a near real feel for design and engineering students and relevant research groups and academicians. It concentrates on disseminating know-how Anthropometric Techniques online. Thus it was proposed to take a tutorial approach and to develop a virtual laboratory manual. Along with the basic principles of anthropometry and its practical experimentation (simulated tutorials) the whole package was uploaded over the net; <iitg.vlab.co.in>, other laboratories, design \& humanities section. This can be used free of cost by anybody interested (specifically targeting Indian users) is expected to serve a ready reference with India relevant anthropometric data support [2]. The virtual ergonomics laboratory imparts knowledge and know-how techniques of human body measurements and ergonomics assessments in virtual reality appears to be appropriate in today's context. This package is expected to bring a know-how resource material.

The input applications allow users to experience a virtual tour to human physical dimension (static as well as varied dynamic) relevance in design and practice the evaluation of a workplace scenario, assisted by a checklist specifying the minimum ergonomic requirements. By acquiring such virtual experience, it would be easier to conduct real life experiments and data collection for specific requirement later.

\section{Target group who can use this online facility: UG, PG general input or Research?}

The importance of the subject is recognised [3], but lacks of laboratory facilities are there to impart the training and easy available ready reference material specific to the Indian context. The developed online virtual manual targets basically the students of UG and PG level as open source material to cater anthropometric issues, design, engineering and architecture. It is expected to serve as a ready manual of human physical- work equipment/ machine- work environment/ space interaction interface for direct learning and for specific research it would assist as ready reference. When the required data source as required to dimension a design one need to generate data as he requires. Considering this need, the package provides the measuring procedure when the ready data pack is not available to use directly and there by statistical treatments and data usage for ergonomics design practice, and thus it expects to cater relevant needs to various practicing as well as research groups.

\section{The content: subject matter introduction and experiments covered}

The content flows flash based simulated tutorial and practical manual through step-by-step measuring procedure, quiz and assignments for self-evaluation, related resources, and additional reference helps. With the help of data set provided here in (Indian human body dimensions in static and dynamic [2]), users are expected to test design and process ideas of good interface. Real experiments were performed in ergonomics laboratory of IIT Guwahati with human subjects in specific contextual setups as supportive background material and video recordings on the same were used for tutorial supplements. Ergonomics for beginners with special reference to industrial 
design perspective, a 40 video lecture of one hour duration each is similar an effort towards disseminating free course material online [4] simultaneously was carried out along with the present virtual laboratory exercise. Target users are expected to go through both for a comprehensive understanding of the subject matter [5].

Title of the present online lab-manual package is "Ergonomics Laboratory for Assessing Physical Aspects of Design", and it contains two sections, i.e., A) introduction to ergonomics which is followed by B) ten topic specific experiments section.

\section{A. Introductory session}

The package begins with an Introduction to ergonomics section which covers the general theory background, methods normally followed to have human body dimensional compatible design practice and varied design application possibilities. This section also discusses the statistical methods; data reference and ergonomic design practice relevance. This section contains 1. Definition, 2. Domains of ergonomics, 3. Human body-Physical dimension, 4. Body planes, 5. Anthropometry, 6. Need for Indian anthropometry, 7. Guidelines for design use, 8. Percentile selection for design use, 9. Use of average and 10. Consideration for regular percentiles. This section is supported with descriptive illustrations to the topic texts and familiarising the basic measuring devices commonly used for anthropometry and general measuring techniques for learning the basics.

\section{B. Experiment section}

This experiment section comprises of ten specific topics, i.e., 1.Static anthropometry, standing heights, 2. Arm forward reaches, standing erect and forward bend, 3. Sitting anthropometry, static heights, 4. Horizontal work surface at around elbow height, 5. Effective work platform height, standing, 6. Effective vertical work surface, 7. Hand dimension, 8. Human dimensional consideration for general seating, 9. Squatting posture, and 10. Body movement ranges.

All the ten experiments follow similar flow and presentation pattern along with cover section which are a) general theory background, b) about experiment-the introductory note followed by reference data sheet, c) practical experience based on simulated presentation of measuring procedure where users can feel the experiment with hands with inserting specific instructions, description, specific experiment setup, equipment/ measuring devices specific to the experiment used and handling technique, measurement procedures, step by step procedure, direct measuring method, indirect (photo-video analysis) method, animated demonstration cum experiments of measurement where users can feel experience (flash based simulation), d) application relevance that cites various application situations, e) some quizzes with simulated multiple choice question answers and self-assessment situations, and f) option for feedback by the users for necessary incorporation and clarification.

It also has lecture support tutorial video. While going through any experiment from any point one can go to anywhere of other experiments for cross reference as one may feel to refer. Users are expected to get the feel of the measuring process through this animated experiment and are advised to measure in real life practical situations using various anthropometric rods, boards etc. sets. Moreover, the users may select their own specific landmarks as required.

Ten specific topic guides and experiments:

Static anthropometry, standing heights: This section focuses on demonstrating various body landmarks, reference data pack and animated measuring procedure. It contains general theory, about experiment, measurement procedures, step by step procedure, direct method, indirect method and animated demonstration of the measuring technique with anthropometer. This followed by reference datasheet relevant to Indian anthropometric dimension in standing posture, i.e., heights, depths, breadths and circumferences at specific landmarks and animated practical experiment with a key information to perform the experiment, and application relevance.

Arm forward reaches, standing erect and forward bend: It describes the comfortable arm reaches in both standing erect and forward bending measurement landmarks- heights and lengths, reference data pack and animated experiments. Relevant theory portion followed by measuring 
procedure in general, step by step procedure for direct measuring and mentions of indirect methods. The datasheet relevant to Indian arm reach dimension in standing posture at lower most, middle and upper most position i.e., heights from floor and length from vertical axis at the heel points is provided as reference. Animated practical to-do-by-self for both stand erect and bend forward consisted the main experiment section, and it follows relevant design application issues.

Sitting anthropometry, static heights: It focuses on sitting height measurement landmarks, reference data pack and animated experiments. It starts with mention of about experiment, measuring procedures, direct methods and indirect methods, and animated demonstrations.

Horizontal work surface at around elbow height: It is focused at understanding various horizontal work surfaces that are in effect in our daily life, may be the office table, kitchen platform, console or any interactive work counter and various work component layout there in.

Effective work platform height, standing: objective of this section is to understand various work surface heights relevant to normal work platform, table, kitchen platform, or any interactive work counters. It is provides with the reference data set in Indian population. This hands on trial animated experiment setup provides a feel of measuring relevant dimensions and it is supported with a video lecture.

Effective vertical work surface: It aims at understanding the various vertical work surfaces exist in our daily life, may be the office table, kitchen platform, console or any interactive work counter and various work component layout there in. The animated figure gives a virtual experience of measuring selective heights where clicking arrow buttons on the figure helps to get a real feel of experimenting.

Hand dimension: This section focuses on hand dimension measurement landmarks, reference data pack and animated experiments. It presents the measuring technique using a 'click the image to perform the experiment' through animated demonstration of caliper which can also be used to measure various hand figures placing on it and measure by clicking the direction buttons on keyboard; the data can also be checked with percentile variations by clicking another button. It has a tutorial video support also on the topic.

Human dimensional consideration for general seating: The session focuses on anthropometric issues for seating and relevant details specifically supported with animated demonstration with clicking options to get relevant dimensions with varied postural considerations and video tutorials demonstrating the seating in various context and human dimensions required to support the same.

Squatting posture: This experiment gives an understanding the relevant space or distance required to perform different task in squatting posture (specifically in Indian work context). The experiment gives a feeling of real experiment to perform task in actual and or simulate situations. The experiment follows "click the image and press up arrow and down arrow to move the measuring scale (anthropometer counter) on the human figure. Squatting posture while performing activities and some design concepts are given here to acquaint with the issues and this section is supported with a video tutorial.

Body movement ranges: This experiment focuses on different body movements mainly flexion, extension of different parts of the body with special emphasis on head, neck, trunk, arm, hip, and leg. Animated experiment is based on goniometer and follows 'drag the hand and place the joints at the center of the goniometer and measure the angle, click the arrow buttons below to move the scale of the goniometer'. To acquaint with the issue it provides visual references of body movement ranges and workstation design which can be harmful to the workers and considerations of a car driver.

\section{Development process, time frame followed and target users' feedback}

It is a three-year effort. $1^{\text {st }}$ year of the project was devoted to plan the total project scope, identifying the need based areas/ topics to be documented; experts opinion have been collected; getting equipped with relevant information, software procurement; and creating experimental set-ups. Experiments were conducted in the laboratory and field conditions. Audio-visual recordings were made to express the techniques and aspects. Virtualisation of few experiments were tried out and 
shown to the students groups at IIT Guwahati and others from selective academic institutes dealing with ergonomics for feedback.

$2^{\text {nd }}$ year was committed to make the full set of experiments as per objectives and ten specific topics as mentioned above. Flash based simulations suitable for virtual hands-on experiences of the experiments and relevant video demonstrations of real experiments and on the same constituted the content.

$3^{\text {rd }}$ year, the ongoing period concentrates on field trials and refinement modification; final AV manual was prepared for online presentation. It is uploaded using IIT Guwahati server. Feedback received online from likely to be users groups and while demonstrating in person has been incorporated to refine the content. Now it is ready for final go.

Intermittent meetings with experts and beneficiaries (selected students groups and professionals) were carried out. Seminars and workshops on the development process took place. The manual thus prepared is expected to serve as a self-explaining lab-manual. As it is expected to be useful to the teaching community and students to imparting training and relevant professionals for useful data collection methods and reference guidelines on measuring techniques they will be able to perform the experiments in the real lab and field situations.

While carrying out the development process, students groups and faculty members from architecture students at Guwahati, Assam and Masters' degree of ergonomics specialisation students of Calcutta University, Vidyasagar University and Kalyani University of West Bengal constituted the participatory representation of users groups. The package was also demonstrated in preconference workshop of Indian society of ergonomics conducted annual conferences held at IIT Madras where a cross section of participants ranging from agricultural engineering to family resource management, architecture, engineering, industrial design, ergonomics specialists and industry were present. Design students of IIT Guwahati of both UG and PG of last three years were taken into confidence to have the main feedback group.

The ergonomics virtual lab exercise was conceived as one of the component projects of a national coordinated project of virtual laboratory for technical disciplines in India. Intermittent development and feedback incorporations were constantly checked by presenting before the expert group (nominated by the apex body of the main project) comprising different subject expertise (non-ergonomics); thus the package confirms users' comfortable flow to even to the non-subject expert users.

\section{Conclusion: does it replace the need of 'real lab'? - The expected outcome (final deliverables)}

Students, may visit web uploaded cite or using CD, are expected to become familiar of ergonomics aspects and experiment demonstrations presented there in. Simulated experiments they can practice of their own and that finally would assist in conducting direct real lab and field experiments. The material made available as an outcome of this project is self-sustaining, no need for specialised equipment or software to read and use the manual.

Expected outcome of this package is a Lab-Manual (animated) and related information material, which is going to be uploaded soon in a designated national website to share the information to the intended beneficiaries. Interactive audio-visual presentation for academic use (design, engineering, management and ergonomics and relevant) may also be used by the professionals as references. It gives confidence to plan for similar programmes to suit various discipline requirements with audiovisual supportive material for non-contact training and for hands on experiment at IIT Guwahati. 


\section{References}

[1] P. Kumar and D. Chakrabarti, 'User Centered design input in Mechanical Engineering and Design: Ergonomics relevance', Proc. International Ergonomics Conference (Humanising Work and Working Environment), Indian Society of Ergonomics conference, Calcutta University, Kolkata, 17-19 December, 2009.

[2] D. Chakrabarti, Indian Anthropometric dimensions for ergonomic design practice, National Institute of Design, Paldi, Ahmedabad 380007, ISBN No: 81-86199-15-0, 161p, 1997.

[3] D. Chakrabarti, Design relevant research leading to PhD: IIT Guwahati experiences, research into design: Supporting Multiple Factors of product Development, edited by A. Chakrabarti, Proc. in international Conference on research into Design,ICoRD'09, Bangalore), Research Publishing, Singapore- Chennai, ISBN 978-981-08- 2277-4, Pp. 595-599, 2009.

[4] D. Chakrabarti, Ergonomics for beginners: industrial design perspective (an online series of 40 video lectures course material of one hour duration each), NPTEL programme, Indian Institute of Technology Guwahati, http://nptel.iitm.ac.in, 2012.

[5] Sanjog, J., Karmakar, S., Patel, T. and Chowdhury, A., 2012. DHM an aid for virtual ergonomics of manufacturing shop floor: A review with reference to industrially developing countries. International Journal of Computer Applications, (doi: 10.5120/8634-2541) 54(14), 1823. 